\title{
Anti-glaucoma potential of hesperidin in experimental glaucoma induced rats
}

\author{
Baiyang Lu, Xue Wang, Zengjin Ren, Haitao Jiang and Bingqian Liu*
}

\begin{abstract}
Glaucoma is well-known clinical eye conditions that damage the optic nerve due to abnormal pressure conditions in eye. Hesperidin is well-known glycoside widely present in the citrus fruits, and its aglycone form is known as hesperetin. Hesperidin is major flavone found in orange fruits. Hypotensive effect of hesperidin in acute and chronic glaucoma rats, glutamate level in vitreous humour and glutathione (GSH) level in aqueous humour were determined following 25,50 and $100 \mathrm{mg} / \mathrm{kg}$ of hesperidin treatment. Acetazolamide $(5 \mathrm{mg} / \mathrm{kg}$ ) was used as positive control. Hesperidin treatment significantly reduced the increased intraocular pressure (IOP) level in dextrose induced ocular hypertension than saline treated rats. The effect of hesperidin was comparable to the positive control acetazolamide. Similarly, hesperidin treatment significantly reduced the IOP level in prednisolone acetate induced ocular hypertension than saline treated rats. In the aqueous humour, hesperidin treatment increased the glutathione level 125\%, $184.4 \%$ and $231.2 \%$ at 25,50 and $100 \mathrm{mg} / \mathrm{kg}$ of hesperidin respectively. In the vitreous humour, hesperidin treatment reduced the glutamate level $9.9 \%, 13.2 \%$ and $25.3 \%$ at 25,50 and $100 \mathrm{mg} / \mathrm{kg}$ of hesperidin respectively. Histopathological analysis of normal saline treated rats showed morphological alteration in ciliary bodies. However, rats treated with hesperidin showed the reduced level of morphological alteration in ciliary bodies. Taking all these data together, it is suggested that the hesperidin supplementation was effective against glaucoma in experimental rats.
\end{abstract}

Keywords: Hesperidin, Glutathione, Glutamate, Hypertension, Rats

\section{Introduction}

Glaucoma is well-known clinical eye conditions that damage the optic nerve due to abnormal pressure conditions in eye (Kingman 2004). Glaucoma is one of the major factors for irreversible blindness around the world over the age above 60 (Gossman et al. 2016). Researchers have reported that the vascular dysregulation and increased intraocular pressure (IOP) are major causative factors for glaucoma (Kyei et al. 2015), and these factors induces initial injury in the retinal glial cells (Chong and Martin 2015). Researchers have reported that the oxidative damage and injured neurons produced glycine

\footnotetext{
*Correspondence: FredaCararYpRsT@yahoo.com

Department of Ophthalmology, The First Affiliated Hospital

of Kangda College of Nanjing Medical University, Xuzhou Medical

University Affiliated Hospital of Lianyungang, The First People's

Hospital of Lianyungang, No. 6 Zhenhua East Road, Haizhou District,

Lianyungang 222002, China
}

and glutamate that induces excitotoxicity (Pose-Utrilla et al. 2017). Laser and incisional surgery, and medicines are available options for the management of glaucoma (Schwartz and Budenz 2004). Even though, several therapeutic agents available for treating glaucoma, still around $10 \%$ people suffering from the blindness all around the world (Acton 2013).

Researchers have reported that the polyphenols are major research area in the two decades due to their antioxidant potential (Tsao 2010; Gabriele et al. 2017). Several pharmacological and protective effects of polyphenols against cancer, degenerative disease, osteoporosis and cardiovascular disease (Scalbert et al. 2005a, b). Hesperidin is well-known glycoside widely present in the citrus fruits, and its aglycone form is known as hesperetin (Man et al. 2019). Hesperidin is major flavanone found in orange fruits (Kobayashi et al. 2008). Researchers have reported that the various therapeutic and protective effects of hesperidin against cerebral thrombosis,
Springer Open (c) The Author(s) 2020. This article is licensed under a Creative Commons Attribution 4.0 International License, which permits use, sharing, adaptation, distribution and reproduction in any medium or format, as long as you give appropriate credit to the original author(s) and the source, provide a link to the Creative Commons licence, and indicate if changes were made. The images or other third party material in this article are included in the article's Creative Commons licence, unless indicated otherwise in a credit line to the material. If material is not included in the article's Creative Commons licence and your intended use is not permitted by statutory regulation or exceeds the permitted use, you will need to obtain permission directly from the copyright holder. To view a copy of this licence, visit http://creativeco mmons.org/licenses/by/4.0/. 
hypertension, fatty liver, osteoporosis, hypercholesterolemia and asthma (Qian et al. 2016; Chiba et al. 2014; Ikemura et al. 2012; Wei et al. 2012). However, there are no detailed studies available on the protective effect of hesperidin against glaucoma. Thus, we carried out the anti-glaucoma potential of hesperidin in experimentallyinduced glaucoma model of rats.

\section{Materials and methods Chemicals}

Acetazolamide (A6011, Sigma-Aldrich, China) was used as positive control for glaucoma. Proparacaine hydrochloride (1571001, Merck, China) was used as local anaesthetic ophthalmic solution. Prednisolone acetate (1556008, Merck, China) was used to induce ocular hypertension.

\section{Rats}

Male albino rats were obtained from the Animal House of Xuzhou Medical University Affiliated Hospital of Lianyungang, China. The rats (170-200 g) were maintained in rat ages with standard atmospheric conditions of $12 \mathrm{~h}$ light and dark periods at $25 \pm 0.5{ }^{\circ} \mathrm{C}$ with a relative humidity of $60 \pm 5 \%$. All methods were carried out in accordance with relevant guidelines and regulations, and approved by ethical committee of Xuzhou Medical University Affiliated Hospital of Lianyungang, China.

\section{Determination of hypotensive effect of hesperidin in acute glaucoma rats}

The basal IOP levels in the eyes of rat were measured according to previously described method (Pang et al. 2005). A proper care was given to prevent nictitating membrane from arising under tonometer. The weights $5.5 \mathrm{~g}$ and $10 \mathrm{~g}$ was used to induce tension, and recorded. The mean value of both recordings was determined. Then, rats were grouped into normal saline (group I), acetazolamide (5 mg/kg, group II), $25 \mathrm{mg} / \mathrm{kg}$ of hesperidin (group III), $50 \mathrm{mg} / \mathrm{kg}$ of hesperidin (group IV) and $100 \mathrm{mg} / \mathrm{kg}$ of hesperidin (group V). All the doses were given to rats through oral gavage, and dose volume adjusted to $1 \mathrm{ml}$. Following $30 \mathrm{~min}$ of dose treatment, rats were administered dextrose solution $(5 \% ; 15 \mathrm{ml} / \mathrm{kg}$ ) via marginal ear vein. The IOP value was measured for $2 \mathrm{~h}$ in every $20 \mathrm{~min}$.

\section{Determination of ocular hypotensive effect of hesperidin in chronic glaucoma rats \\ Experimental model of ocular hypertension}

The ocular hypertension was induced in rats through instilling prednisolone acetate (1\%) in the eyes following baseline IOP determination two times per day, and it was continued for 21 days. The IOP level was measured in weekly basis between 8.30 and 9.00 AM. Symptoms such as fixed dilated pupils, limbal injection, bulging eyeball and limbal injection were observed in the rats having at least 50\% increase in IOP (Dey et al. 2018).

\section{Determination of ocular hypertension effect of hesperidin}

Rats with ocular hypertension were grouped into normal saline (group I), acetazolamide $(5 \mathrm{mg} / \mathrm{kg}$, group II), $25 \mathrm{mg} / \mathrm{kg}$ of hesperidin (group III), $50 \mathrm{mg} / \mathrm{kg}$ of hesperidin (group IV) and $100 \mathrm{mg} / \mathrm{kg}$ of hesperidin (group V). All the doses were given to rats through oral gavage, and dose volume adjusted to $1 \mathrm{ml}$. The dose given to rats for 21 consecutive days, and intraocular pressure was determined in all the eyes for every day (Dey et al. 2018).

\section{Determination of glutamate}

In the vitreous humour, glutamate level was determined by using commercial kit (ab83389, glutamate assay kit, Abcam, UK). Briefly, vitreous humour was collected in the sterile tube and vitreous bodies were sonicated by using perchloric acid $(0.2 \mathrm{M})$. Then, the homogenate was collected and centrifuged at 15,000 $\mathrm{g}$ at cold temperature for $5 \mathrm{~min}$, and then supernatant was collected for the determination of glutamate level. The standards and samples were prepared according to the instruction given in kit, and pipetted in a 96-well plate (Gao et al. 2018). The final absorbance was read at $405 \mathrm{~nm}$ in UV-VIS Spectrophotometer (Shimatzu, Guangdong, China).

\section{Determination of glutathione}

In aqueous humour, the total glutathione was determined according to the instruction given in commercial kit (CS0260, glutathione assay kit, Merck, China). Anterior chamber was punctured by using 30 -gauge needle after euthanizing the rats. Then, aqueous humour from all the eyes were collected in sterile tube. The triethanolamine (4 M) and metaphosphoric acid was used for the deproteining the aqueous humour. Finally, standards and aqueous humour samples were prepared according to the instruction given in kit, and pipetted in a 96-well plate, and incubated for $15 \mathrm{~min}$ at dark place (Iskusnykh et al. 2013). The final absorbance was read at $405 \mathrm{~nm}$ in UVVIS Spectrophotometer (Shimadzu, Guangdong, China).

\section{Histopathological study}

Enucleated eyes of rats were carefully removed in paraformaldehyde (10\%), and then embedded in paraffin. Then, paraffin embedded eyes were sectioned and stained with haematoxylin and eosin. Then, stained sections were fixed in microscopic slides for the examination (Shibuya et al. 2015). The pathological examination was carried out by two independent pathologist at Xuzhou Medical University Affiliated Hospital of Lianyungang, China. 


\section{Statistical analysis}

Data are expressed as mean \pm standard error of the mean (SEM). The groups were compared using Student's $t$-tests and an analysis of variance (ANOVA). $P$ values $<0.05$ were considered to indicate statistical significance.

\section{Results}

Hesperidin treatment significantly reduced the IOP level in dextrose induced ocular hypertension than saline treated rats (group I, $\mathrm{P}<0.05$; Fig. 1). The effect of hesperidin was comparable to the positive control acetazolamide, and there was no significant difference observed between hesperidin and acetazolamide (Fig. 1). Similarly, hesperidin treatment significantly reduced the IOP level in prednisolone acetate induced ocular hypertension than saline treated rats (group I, $\mathrm{P}<0.05$; Fig. 2). The effect of hesperidin was comparable to the positive control acetazolamide, and there was no significant difference observed between hesperidin and acetazolamide (Fig. 1).

Hesperidin treatment prevented the oxidative stress through the recovery of glutathione content. In the aqueous humour, hesperidin treatment increased the glutathione level $125 \%, 184.4 \%$ and $231.2 \%$ at 25,50 and $100 \mathrm{mg} / \mathrm{kg}$ of hesperidin respectively $(\mathrm{P}<0.05$; Fig. 3). Acetazolamide treatment increased the glutathione level 259.3\% ( $<<0.05$; Fig. 3). In the vitreous humour, hesperidin treatment reduced the glutamate level 9.9\%, 13.2\% and $25.3 \%$ at 25,50 and $100 \mathrm{mg} / \mathrm{kg}$ of hesperidin respectively $(\mathrm{P}<0.05$; Fig. 4). Acetazolamide treatment reduced the glutamate level 25.3\% ( $\mathrm{P}<0.05$; Fig. 4).

Histopathological analysis of normal saline treated rats showed morphological alteration in ciliary bodies. However, rats treated with hesperidin showed the reduced level of morphological alteration in ciliary bodies (Fig. 5). Acetazolamide treatment also showed the reduced level of morphological alteration in ciliary bodies (Fig. 5).

\section{Discussion}

Glaucoma is well-known clinical eye conditions that damage the optic nerve due to abnormal pressure conditions in eye (Kingman 2004). Glaucoma is one of the major factors for irreversible blindness around the world over the age above 60 (Gossman et al. 2016). Researchers

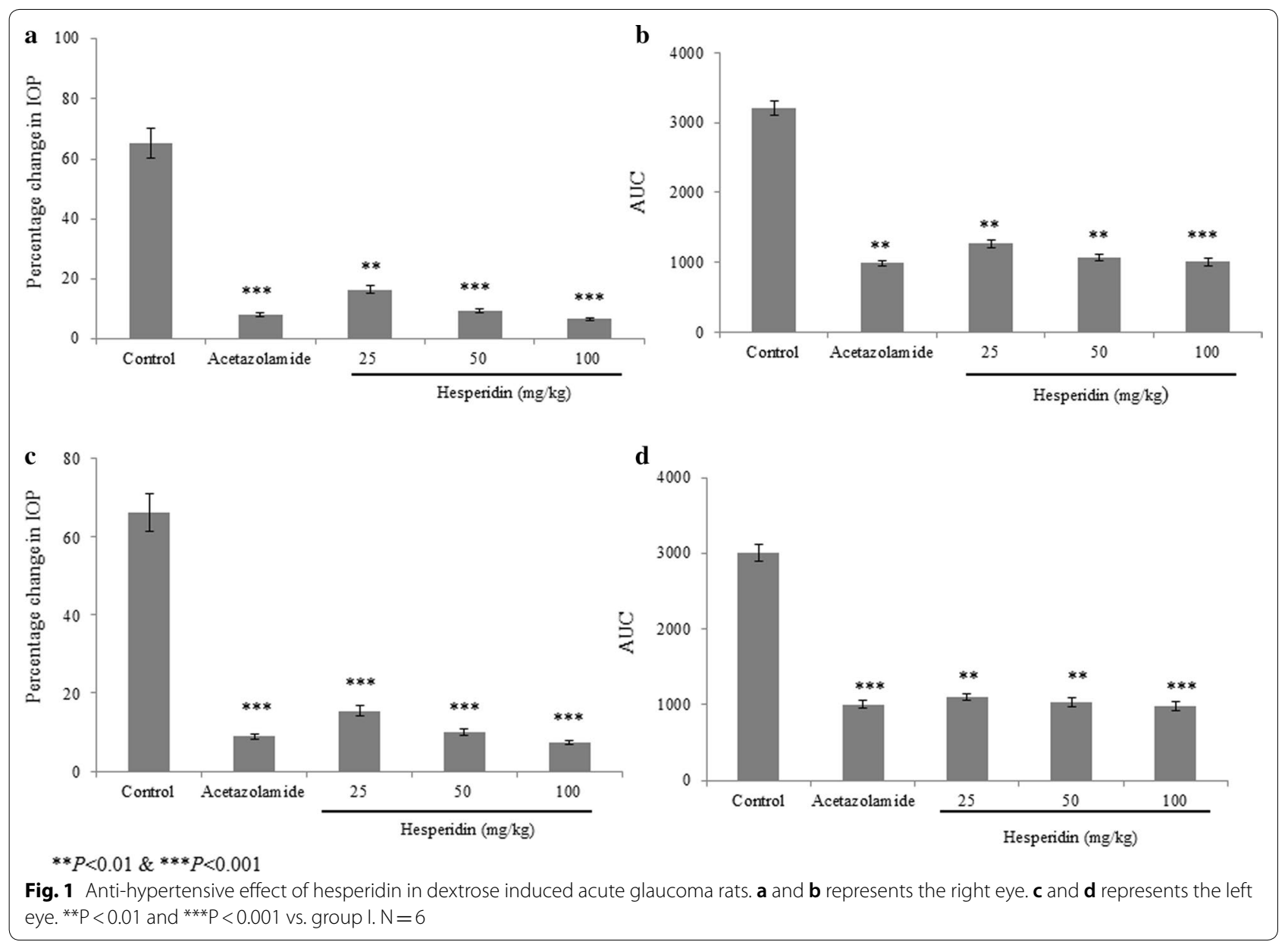




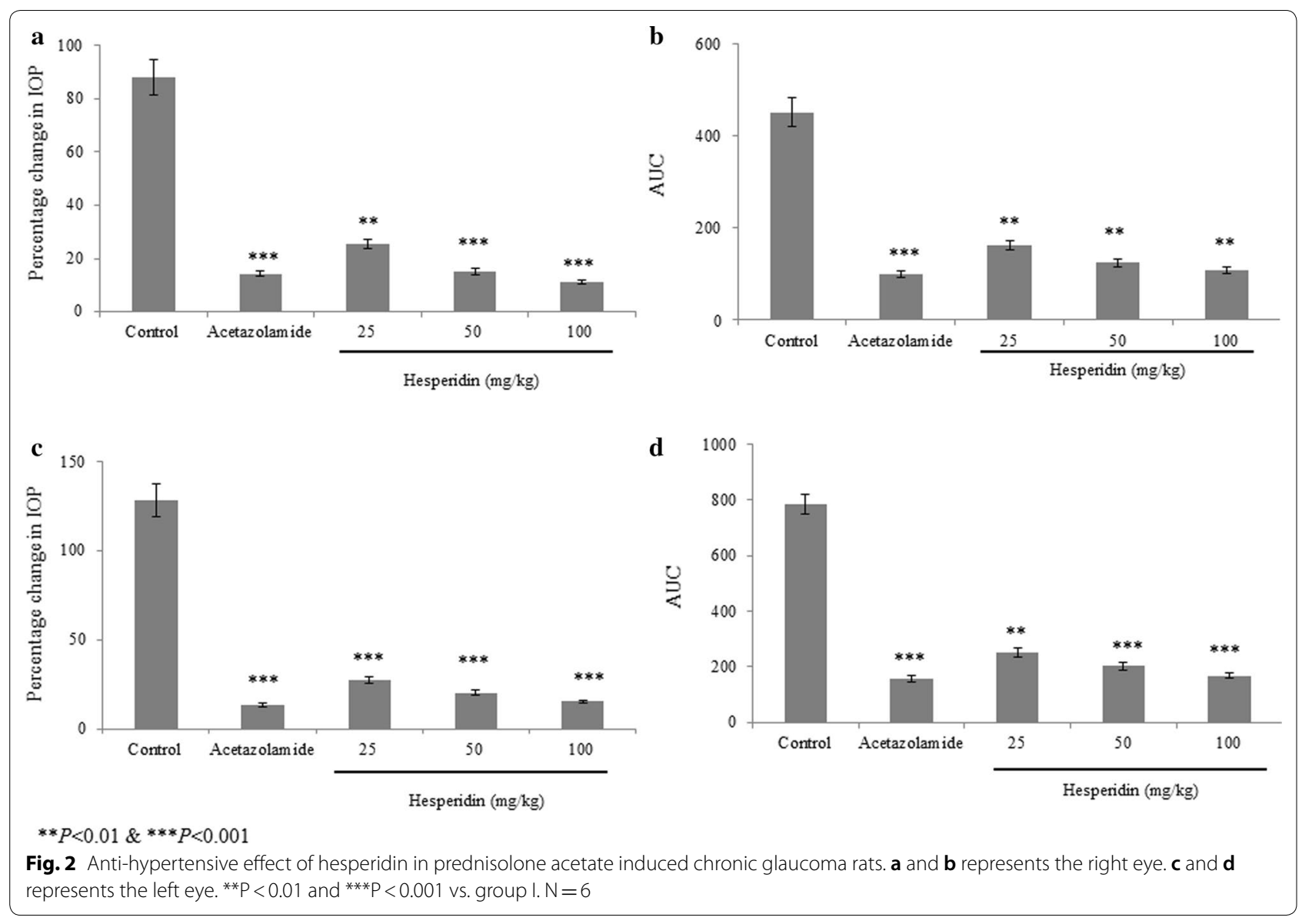

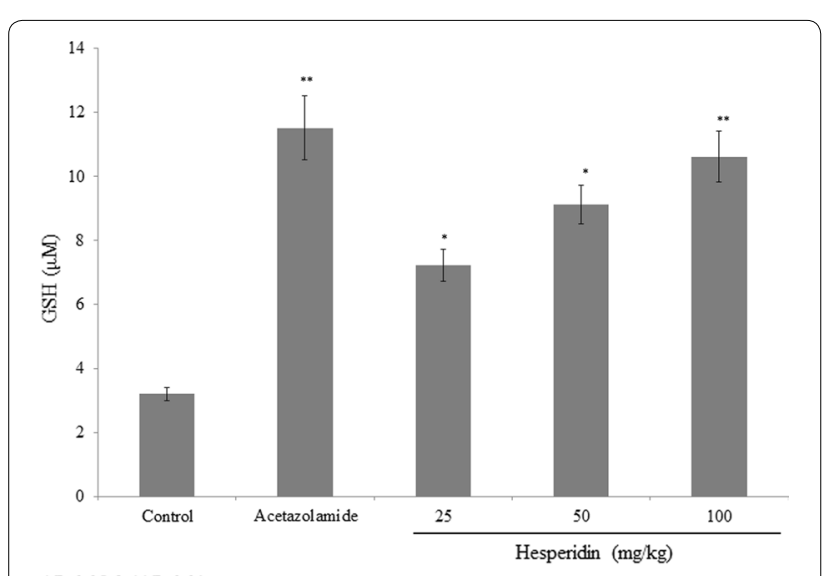

${ }^{*} P<0.05 \& * * P<0.01$

Fig. 3 Protective effect of hesperidin on glutathione level in prednisolone acetate induced chronic glaucoma rats. ${ }^{* *} \mathrm{P}<0.01$ and ${ }^{* * * P}<0.001$ vs. group I. $N=6$

have reported that the vascular dysregulation and IOP are major causative factors for glaucoma (Kyei et al. 2015), and these factors induces initial injury in the retinal glial cells (Chong and Martin 2015). Researchers have

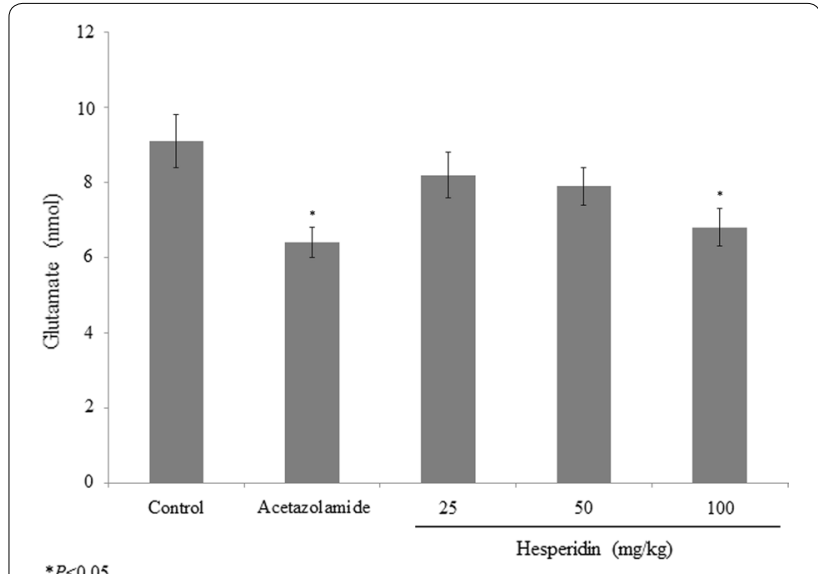

Fig. 4 Protective effect of hesperidin on glutamate level in prednisolone acetate induced chronic glaucoma rats. ${ }^{*} \mathrm{P}<0.01$ and ***P $<0.001$ vs. group I. $N=6$

reported that the oxidative damage and injured neurons produced glycine and glutamate that induces excitotoxicity (Pose-Utrilla et al. 2017). Laser and incisional surgery, and medicines are available options for the management 

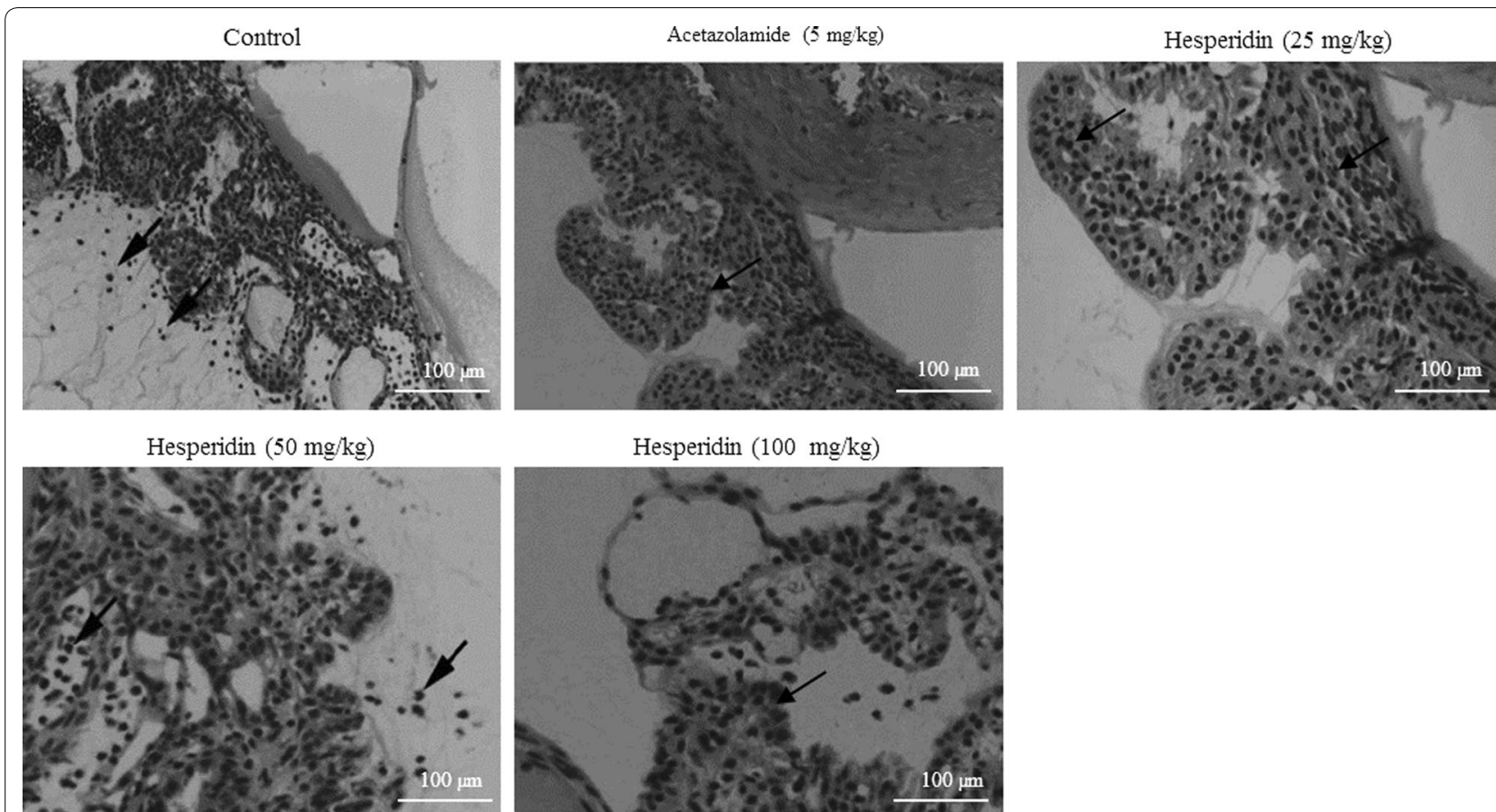

Fig. 5 Histopathological analysis of anterior chamber in prednisolone acetate induced chronic glaucoma rats. $N=6$. Scale bar is $100 \mu \mathrm{m}$

of glaucoma (Schwartz and Budenz 2004). Even though, several therapeutic agents available for treating glaucoma, still around $10 \%$ people suffering from the blindness all around the world (Acton 2013).

Flavonoids are potential supplements for animal studies due to their lower toxicity at higher doses and prolonged period of treatment duration. In this study, we selected hesperidin due to their strong antioxidant potential. Researchers have reported that the various therapeutic and protective effects of hesperidin against cerebral thrombosis, hypertension, fatty liver, osteoporosis, hypercholesterolemia and asthma (Qian et al. 2016; Chiba et al. 2014; Ikemura et al. 2012; Wei et al. 2012). Menze et al. (2012) have reported the antioxidant activity of hesperidin like vitamin. Meloni (1995) have reported anti-inflammatory activity of hesperidin.

Kara et al. (2014) have reported the therapeutic effect of hesperetin against apoptosis in ischemia/reperfusion-induced retinal injury model of rats. Maekawa et al. (2017) have reported the neuroprotective effect of hesperidin in NMDA-induced retinal injury. Therapeutic potential of bioflavonoids against ocular disorders have been reported in several research (Majumdar and Srirangam 2010). Estruel-Amades et al. (2019) have reported the therapeutic potential of hesperidin against oxidative stress in rats. Anti-glaucoma effect of hesperidin was clearly established by evaluating the ocular hypotensive effect in chronic ocular hypertensive model.
Researchers have reported the frequent intake of steroids induces oxidative stress, which leads to aqueous humour antioxidant system and cell loss of apoptotic trabecular meshwork. This, further leads to mitochondrial damage and mitochondrial dysfunction (Saccà et al. 2015). Saccà et al. (2011) have reported the reduction of aqueous production leads to reduced oxidative damage in trabecular meshwork and active mitochondria. Taking all these data together, it is suggested that the hesperidin supplementation was effective against glaucoma in experimental rats.

\section{Abbreviations}

IOP: Increased intraocular pressure; GSH: Glutathione; SEM: Standard error of the mean; ANOVA: Analysis of variance.

\section{Acknowledgements}

None.

\section{Authors' contributions}

$\mathrm{BL}, \mathrm{XW}$ and $\mathrm{ZR}$ conducted experiments and collected data. $\mathrm{HJ}$ and $\mathrm{BL}$ carried out data interpretation, review of literature and manuscript drafting. All authors read and approved the final manuscript.

\section{Funding \\ None.}

Availability of data and materials

Corresponding author could provide the all experimental data on valid request.

Ethics approval and consent to participate

All animal experiments were approved by the ethical committee of the Xuzhou Medical University Affiliated Hospital of Lianyungang, China. 


\section{Consent to participate}

Not applicable.

\section{Consent for publication}

Not applicable.

\section{Competing interests}

Authors declare that they have no conflict of interest.

Received: 15 February 2020 Accepted: 8 May 2020

Published online: 18 May 2020

\section{References}

Acton AQ (2013) Open-angle glaucoma: new insights for the healthcare professional, 2013th edn. Scholarly Editions, Georgia, p 2013

Chiba H, Kim H, Matsumoto A, Akiyama S, Ishimi Y, Suzuki K, Uehara M (2014) Hesperidin prevents androgen deficiency-induced bone loss in male mice. Phyther Res 28:289-295

Chong RS, Martin KR (2015) Glial cell interactions and glaucoma. Curr Opin Ophthalmol 26:73-77

Dey A, Manthey AL, Chiu K, Do CW (2018) Methods to induce chronic ocular hypertension: reliable rodent models as a platform for cell transplantation and other therapies. Cell Transplant 27(2):213-229

Estruel-Amades S, Massot-Cladera M, Garcia-Cerdà P, Pérez-Cano FJ, Franch A, Castell M, Camps-Bossacoma M (2019) Protective effect of hesperidin on the oxidative stress induced by an exhausting exercise in intensively trained rats. Nutrients. https://doi.org/10.3390/nu11040783

Gabriele M, Frassinetti S, Caltavuturo L, Montero L, Dinelli G, Longo V, Di Gioia D, Pucci L (2017) Citrus bergamia powder: antioxidant, antimicrobial and anti-inflammatory properties. J Funct Foods 31:255-265

Gao L, Zheng QJ, Ai LQ (2018) Exploration of the glutamate-mediated retinal excitotoxic damage: a rat model of retinal neurodegeneration. Int J Ophthalmol 11:1746-1754

Gossman CA, Linn DM, Linn C (2016) Glaucoma-inducing procedure in an in vivo rat model and whole-mount retina preparation. J Vis Exp 12(109):e53831

Ikemura M, Sasaki Y, Giddings JC, Yamamoto J (2012) Preventive effects of hesperidin, glucosyl hesperidin and naringin on hypertension and cerebral thrombosis in stroke-prone spontaneously hypertensive rats. Phyther Res 26:1272-1277

Iskusnykh IY, Popova TN, Agarkov AA, Pinheiro de Carvalho MÂ, Rjevskiy SG (2013) Expression of glutathione peroxidase and glutathione reductase and level of free radical processes under toxic hepatitis in rats. J Toxicol 2013:870628

Kara S, Gencer B, Karaca T, Tufan HA, Arikan S, Ersan I, Karaboga I, Hanci V (2014) Protective effect of hesperetin and naringenin against apoptosis in ischemia/reperfusion-induced retinal injury in rats. Sci World J 2014:797824

Kingman S (2004) Glaucoma is second leading cause of blindness globally. Bull World Health Organ 82:887-888

Kobayashi S, Tanabe S, Sugiyama M, Konishi Y (2008) Transepithelial transport of hesperetin and hesperidin in intestinal Caco-2 cell monolayers. Biochim. Biophys Acta Biomembr 1778:33-41
Kyei S, Koffuor GA, Ramkissoon P (2015) Anti-glaucoma potential of Heliotropium indicum Linn in experimentally-induced glaucoma. Eye Vis 2:16

Maekawa S, Sato K, Fujita K, Daigaku R, Tawarayama H, Murayama N, Moritoh S, Yabana T, Shiga Y, Omodaka K, Maruyama K, Nishiguchi K, Nakazawa T (2017) The neuroprotective effect of hesperidin in NMDA-induced retinal injury acts by suppressing oxidative stress and excessive calpain activation. Sci Rep. https://doi.org/10.1038/s41598-017-06969-4

Majumdar S, Srirangam R (2010) Potential of the bioflavonoids in the prevention/treatment of ocular disorders. J Pharm Pharmacol 62(8):951-965

Man MQ, Yang B, Elias PM (2019) Benefits of hesperidin for cutaneous functions. Evid Based Complement Altern Med 2019:2676307

Meloni F (1995) Effects of 3'-hydroxyfarrerol (IdB 1031), a novel flavonoid agent, on phagocyte products. Inflammation 19:689-699

Menze ET, Tadros MG, Abdel-Tawab AM, Khalifa AE (2012) Potential neuroprotective effects of hesperidin on 3-nitropropionic acid-induced neurotoxicity in rats. Neurotoxicology 33:1265-1275

Pang $\mathrm{IH}$, Wang WH, Clark AF (2005) Acute effects of glaucoma medications on rat intraocular pressure. Exp Eye Res 80:207-214

Pose-Utrilla J, García-Guerra L, Del Puerto A, Martín A, Jurado-Arjona J, De León-Reyes NS, Gamir-Morralla A, Sebastián-Serrano Á, García-Gallo M, Kremer L, Fielitz J, Ireson C, Pérez-Álvarez MJ, Ferrer I, Hernández F, Ávila J, Lasa M, Campanero MR, Iglesias T (2017) Excitotoxic inactivation of constitutive oxidative stress detoxification pathway in neurons can be rescued by PKD1. Nat Commun 8(1):2275

Qian W, Hasegawa J, Cai X, Yang J, Ishihara Y, Ping B, Tsuno S, Endo Y, Matsuda A, Miura N (2016) Effects of hesperidin on the progression of hypercholesterolemia and fatty liver induced by high-cholesterol diet in rats. Yonago Acta Med 59:67-80

Saccà SC, La Maestra S, Micale RT, Larghero P, Travaini G, Baluce B (2011) Ability of dorzolamide hydrochloride and timolol Maleate to target mitochondria in glaucoma therapy. Arch Ophthalmol 129:48-55

Saccà SC, Pulliero A, Izzotti A (2015) The dysfunction of the trabecular meshwork during glaucoma course. J Cell Physiol 230:510-525

Scalbert A, Manach C, Morand C, Rémésy C (2005a) Dietary polyphenols and the prevention of diseases. Crit Rev Food Sci Nutr 45:287-306

Scalbert A, Johnson IT, Saltmarsh M (2005b) Polyphenols: antioxidants and beyond. Am J Clin Nutr 81:215S-217S

Schwartz K, Budenz D (2004) Current management of glaucoma. Curr Opin Ophthalmol 15(2):119-126

Shibuya K, Tomohiro M, Sasaki S, Otake S (2015) Characteristics of structures and lesions of the eye in laboratory animals used in toxicity studies. J Toxicol Pathol 28(4):181-188

Tsao R (2010) Chemistry and biochemistry of dietary polyphenols. Nutrients 2:1231-1246

Wei D, Ci X, Chu X, Wei M, Hua S, Deng X (2012) Hesperidin suppresses ovalbumin-induced airway inflammation in a mouse allergic asthma model. Inflammation 35:114-121

\section{Publisher's Note}

Springer Nature remains neutral with regard to jurisdictional claims in published maps and institutional affiliations.

\section{Submit your manuscript to a SpringerOpen ${ }^{\circ}$ journal and benefit from:}

- Convenient online submission

- Rigorous peer review

- Open access: articles freely available online

- High visibility within the field

Retaining the copyright to your article

Submit your next manuscript at springeropen.com 